\title{
Peculiar Metal Poor Stars as Guides to Key Processes in the Early Halo
}

\author{
Johannes Andersen ${ }^{1,2}$ and Birgitta Nordström ${ }^{1}$ \\ ${ }^{1}$ Niels Bohr Institute, University of Copenhagen, \\ Juliane Maries Vej 30, DK-2100, Copenhagen, Denmark \\ email: ja, birgitta@nbi.ku.dk \\ ${ }^{2}$ Nordic Optical Telescope, Apartado 474, Santa Cruz de La Palma, Spain \\ email: ja@not.iac.es
}

\begin{abstract}
Large photometric or spectroscopic surveys are used to sort stars into populations and define the main trends that characterise them, as diagnostics of their origin. Stars falling off the trends defined by the 'normal' stars are called 'peculiar' and typically eliminated in discussions of Galactic structure and evolution. In our programme on extremely metal-poor halo giants, we have recently focused on the small subgroup that is strongly enhanced in $r$ process elements, asking whether the chemical peculiarity is intrinsic to these stars or due to local surface pollution caused by mass transfer from a binary companion. Precise radial-velocity monitoring over several years turns out to disprove the binary hypothesis and has led to new insight in the processes of chemical enrichment in the early Galactic halo. An ongoing analogous programme on carbon-enhanced metal-poor giants is briefly described at the end.
\end{abstract}

Keywords. Galaxy: formation, Galaxy: halo, stars: abundances, Stars: carbon, Stars: chemically peculiar, Stars: abundances, Binaries: general

\section{Introduction}

In surveys designed to investigate Galactic structure, a few simple photometric or spectroscopic criteria are typically used to sort a large initial sample of stars into main classes or populations. Representative subsamples are then examined in more detail to delineate evolutionary trends and unravel the underlying physical mechanism(s). Stars with non-standard parameter combinations are labelled as 'peculiar' and rejected as irrelevant to discussions of Galactic structure, although they may be interesting in the context of stellar astrophysics. Binaries ( $\sim 30 \%$ of all stars), chemically peculiar stars such as Am or Ap stars, or large-amplitude pulsating variables are typical examples.

Much of our own recent research has focused on the class of extremely metal-poor (EMP) halo stars $([\mathrm{Fe} / \mathrm{H}]<-2.5)$ detected in the objective-prism surveys of Beers et al. (1985) and Beers et al. (1992) and discussed and classified by Beers \& Christlieb (2005). The abundance patterns of these stars were first explored with $4 \mathrm{~m}$-class telescopes by McWilliam et al. (1995) and Ryan et al. (1996), later in the large, systematic and homogeneous "First Stars" project led by Roger Cayrel and based on observations with the 8m ESO Very Large Telescope (VLT) and its high-resolution spectrograph UVES.

The precise elemental abundance relations for "normal" EMP stars resulting from this work have been reported in a series of papers, notably Cayrel et al. (2004), Hill et al. (2002) and Bonifacio et al. (2009), and have been discussed extensively in the context of chemical evolution models for the early Galactic halo. Here, however, we aim to discuss recent work on some of the chemically peculiar stars that were purposely left aside in the systematic programme. 


\section{2. $r$-process Enhanced Stars}

Sneden et al. (1994) first discovered an EMP star, CS22892-052, in which all the heaviest $r$-process elements were overabundant by a large factor relative to iron and the lighter elements. Later, Hill et al. (2002) also determined a uranium abundance in a similar star, CS31082-001. The HERES survey (Barklem et al., 2005) added several more specimens to this class, which comprises $2-3 \%$ of halo giants with a metallicity close to $[\mathrm{Fe} / \mathrm{H}]=-3$. Exhaustive abundance analyses of the brightest of these stars have been published (Siqueira Mello et al., 2013, and references listed there). The salient results of these analyses are (i): The remarkably precise internal agreement of the abundance pattern of the heaviest $r$-process elements as a group to that seen in the Solar System, and (ii) that the abundance pattern of elements up to the iron peak agreed precisely with that seen for normal EMP giants. The most extreme $(r$-II $)$ stars have abundance excesses of $[\mathrm{r} / \mathrm{Fe}]>1.0$, while the (more numerous) $r$-I stars have $0.3<[\mathrm{r} / \mathrm{Fe}]<1.0$.

\section{Original Composition or Surface Pollution?}

The abundance patterns of the individual stars have been discussed extensively, primarily in the context of nuclear-physics models for the $r$-process. The actual progenitor sites (core-collapse supernovae? Merging neutron star binaries?) and the processes by which these stars acquired their exceptional composition have not been identified, however. The key question is whether the observed $r$-process abundances reflect the initial composition of the clouds from which the stars formed, or whether these elements were deposited on the surface by mass transfer from a former binary companion which subsequently exploded as a supernova.

The implications are clearly qualitatively different in the two cases: If the large $r$ process excesses were imprinted on the parent clouds before the stars were born, the $r$-process elements must have been produced and ejected non-isotropically in an inhomogeneous early ISM. If the peculiar chemical composition arose purely locally, it can be safely ignored in models of the chemical evolution of the Galactic halo. Qian \& Wasserburg (2001) advocated the latter scenario, invoking the analogy with the $\mathrm{Ba}$ and $\mathrm{CH}$ stars, all of which are known to be long-period binaries. We decided to test this hypothesis observationally.

\section{Radial-Velocity Monitoring Programme @ NOT}

Since 2007 we have monitored the sample of all 17 known $r$-I and $r$-II stars accessible to the $2.5 \mathrm{~m}$ Nordic Optical Telescope (NOT) on La Palma, Canarias, Spain, and its fibrefed bench-mounted echelle spectrograph FIES, which is housed in a thermally stabilised underground vault. $8 r$-I and $9 r$-II stars were observed at a resolution of $R<\sim 45,000$, to the limits $\delta>-27 \mathrm{deg}$ and $V<15.9$. Because our aim was only to determine radial velocities by cross-correlation, the individual spectra have peak $\mathrm{S} / \mathrm{N}$ ratios of $\sim 10$ or lower. The spectra were reduced and analysed with special-purpose software kindly provided by Dr. L. Buchhave. Radial-velocity standard stars were observed every night, and the velocities were found to be stable at the level of $\sim 35 \mathrm{~m} \mathrm{~s}^{-1}$.

As demonstrated in Fig. 1, we have indeed achieved the desired accuracy of 1-200 m $\mathrm{s}^{-1}$ (somewhat lower for the faintest stars) over a time span of $\sim 2000 \mathrm{~d}$. At this level of precision, we are exceedingly unlikely to miss any plausible binary motion.

Our salient results were summarised by Hansen et al. (2011): 14 stars in our sample are found to be single, including the two most extreme $r$-II stars. Two $r$-I stars are classical 

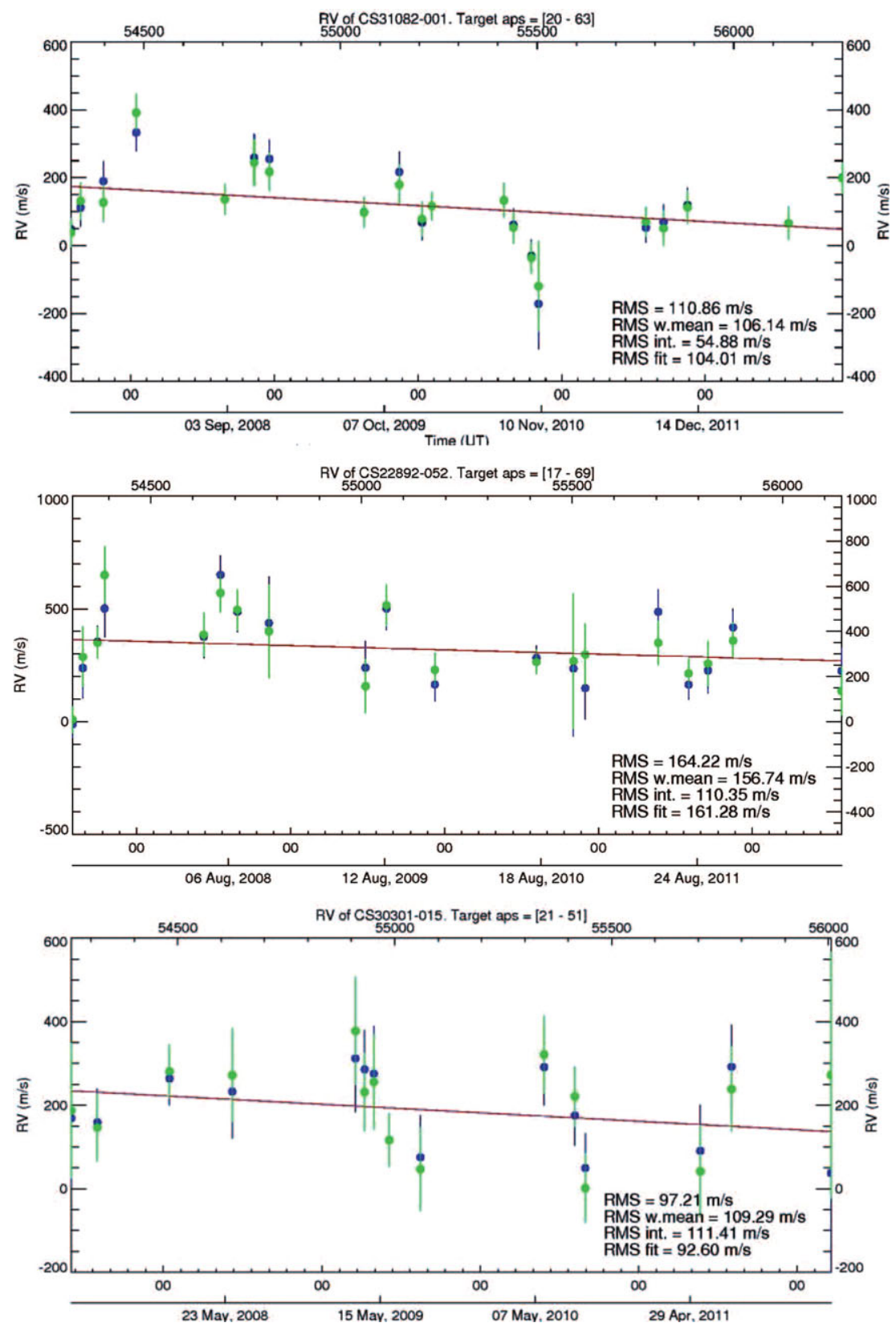

Figure 1. Radial velocities vs. time for three single $r$-process enhanced stars.

spectroscopic binaries with periods of $38 \mathrm{~d}$ and $\sim 2500 \mathrm{~d}$, and have orbital eccentricities typical of spectroscopic binaries among Population I cluster giants Jorissen et al. (1998). A low-amplitude, low-eccentricity orbit with a period of $\sim 300 \mathrm{~d}$ was tentatively found for one of the $r$-II stars; continued monitoring supports the reality of this orbit.

In summary, the binary frequency $(\sim 20 \%)$ and orbital properties among $r$-process enhanced stars are completely normal, and local pollution from a binary companion is ruled out as the general explanation for the $r$-process enhanced EMP giants. 


\section{Implications for Models of the Early Galactic Halo}

The very tightly constrained abundance patterns of 'normal' EMP stars by Cayrel et al. (2004) tell us, first, that most of the envelopes of the first supernovae were ejected roughly isotropically and were well mixed with the ejecta of neighbouring supernova events. Second, the similarly well-defined, but independent abundance pattern of $r$-process elements tells us that the production and ejection of the $r$-process elements was essentially decoupled from those of the lighter elements. Third, whatever the nature of the actual $r$-element production site(s) - core-collapse supernovae or merging neutron star binaries have both been proposed - the freshly produced neutron capture elements were deposited nonisotropically in a small fraction of the clouds in an inhomogeneous early ISM. $r$-element poor stars like HD122653 would then form from clouds that had not been similarly enriched at an early stage; whether they would be the rule or the exception is difficult to ascertain observationally, as metal-poor stars with abnormally weak lines of rare elements are not as easily spotted in spectroscopic surveys as metal-poor stars with spectacularly strong lines of $\mathrm{Ba}, \mathrm{Eu}$ and other $r$-process elements.

Many open questions remain concerning the details of the $r$-process production sites and the structure of the ISM in the early Galactic halo. However, the realisation that these stars inherited their outstanding chemical anomalies from their parent clouds has placed them in a totally new and far more significant context than merely that of interacting binaries: The peculiar exceptions should not be dismissed summarily; they have a very interesting story to tell!

\section{Carbon enhanced extremely metal-poor stars}

A more common and spectroscopically even more conspicuous peculiarity among extremely metal-poor stars is a strong overabundance of carbon, up to $[\mathrm{C} / \mathrm{Fe}]>+2$ dex or more. These so-called CEMP stars comprise $\sim 20 \%$ of the giant stars below $[\mathrm{Fe} / \mathrm{H}]$ $\sim-2.5$, rising to $\sim 40 \%$ below $[\mathrm{Fe} / \mathrm{H}] \sim-3.5$, and come in two varieties, with or without a parallel enrichment in $s$-process elements (CEMP-s and CEMP-no stars, respectively Beers \& Christlieb, 2005). Some CEMP stars exhibit enhancements of $r$-elements, and some of $s$-process elements as well (CEMP-r/s stars). Recent work has found that the CEMP- $s$ and CEMP-no stars are predominantly associated with the inner and outer halo, respectively (Norris et al. 2013 and references therein).

The traditional explanation for the origin of the CEMP stars is also local surface pollution from a former AGB binary companion, and a substantial fraction of the CEMP$s$ stars has indeed been found to be long-period binaries, often with near-circular orbits. But the large incidence of CEMP stars among the most metal-poor outer-halo population challenges the binary paradigm to the limit, and there is so far no observational proof that all CEMP (and especially CEMP-no) stars are binaries. Alternative theoretical models for the earliest production of carbon suggest massive, fast-rotating near-primordial stars or so-called 'faint' supernovae as the production sites, but again observational confirmation is missing. Given the existence of at least some carbon-enriched DLA systems Cooke et al. (2011) and the potential role of carbon gas or dust in expediting the formation of low-mass stars Frebel et al. (2007), the 'peculiar' CEMP stars are clearly also tracers of important processes in the formation and early evolution of galactic halos that are not yet well understood.

We have therefore recently expanded our radial-velocity survey of chemically peculiar EMP stars with a sizeable sample of CEMP stars. The observations are still ongoing, so no conclusions can be presented here, but indications are that the binary frequency 
is substantially below $100 \%$. However, the available abundance data for the neutroncapture elements is not always very reliable, so more work on the chemical classification of the programme stars is also needed to clarify the picture.

\section{Conclusion}

As discussed elsewhere in this meeting, large surveys of 'normal' stars are needed to reveal large-scale features in the structure and origin of the Galaxy. But some exceptional stars may act as "the Canary bird in the mine" and call attention to previously unrecognised, but significant features of the big picture. A couple of examples have been shown here.

Acknowledgements: This work is based on observations made with the Nordic Optical Telescope, operated by the Nordic Optical Telescope Scientific Association at the Observatorio del Roque de los Muchachos, La Palma, Spain, of the Instituto de Astrofisica de Canarias. We are grateful to the Danish Research Council and to the Carlsberg Foundation for support. Most of the observations were obtained in service mode by NOT students and staff, and PhD student Terese Hansen (now Heidelberg) did most of the data reduction with a software package written by Dr. L. Buchhave.

\section{References}

Barklem, P. S., Christlieb, N., Beers, T. C., et al. 2005, A\&A, 439, 129

Beers, T. C., Preston, G. W., \& Schectman, S. A. 1985, AJ, 90, 2089

Beers, T. C., Preston, G. W., \& Shectman, S. A. 1992, AJ, 103, 1987

Beers, T. C. \& Christlieb, N. 2005, ARAA 43, 531

Bonifacio, P., Spite, M., Cayrel, R., et al. 2009, A\&A, 501, 519

Cayrel, R., Depagne, E., Spite, M., et al. 2004, A\&A 416, 1117

Cooke, R., Pettini, M., Steidel, C.C., et al. 2011, MNRAS 412, 1047

Frebel, A., Christlieb, N., Norris, J. E. et al. 2007, MNRAS, 380, L40

Hansen, T., Andersen, J., Nordström, B., Buchhave, L. A., \& Beers, T. C. 2001 ApJL 743, L1

Hill, V., Plez, B., Cayrel, R., et al., 2002 A\&A 387, 560

Jorissen, A., Van Eck, S., Mayor, M., \& Udry, S. 1998 A $\& A$ 332, 877

McWilliam, A., Preston, G. W., Sneden, C., \& Searle, L. 1995, AJ 109, 2757

Siqueira Mello, C., Spite, M., Barbuy, B. et al. 2013, A\& A, 550, 122

Norris, J. E., Yong, D., Bessell, M. S. et al. 2013, ApJ 762, 28

Ryan, S. G., Norris, J. E., \& Beers, T. C. 1996, ApJ, 471, 254

Sneden, C., Preston, G. W., McWilliam, A., \& Searle, L. 1994, ApJ, 431, L27

Qian, Y.-Z. \& Wasserburg, G. 2001, ApJL, 552, L55

\section{Discussion}

Michael Feast: Are there $r$-process rich stars which are not carbon rich?

Johannes Andersen: Yes; CDS 22892-052 is the exception. The others, including the uranium stars CS31082-001 and HE1523-0901, are not carbon enhanced.

Eline Tolstoy: Do you think that the carbon enrichment could relate to the environment in which these stars formed? Or some other initial condition, which could explain the lack of C-rich stars in dSph galaxies? 
Johannes Andersen: Some prolific producers of C are assumed to be very massive. Depending on the IMF and the total stellar mass, they could be missing, so that AGB stars would only contribute later (i.e. at higher metallicity). But trace amounts of $\mathrm{C}$ (and $\mathrm{O}$ ?) were presumably needed to form low-mass stars at all.

PATRICK DE LAVERnY: Several extremely metal-poor stars of the Galactic halo are found to be very carbon enriched. In contrast, no such C-rich EMP stars are found in dSph galaxies (see. E. Tolstoys talk). Do you have any idea about the cause(s) of such a difference?

Johannes Andersen: Not really. But if the progenitors of the (single) CEMP-no stars in the outer Galactic halo were very massive, they could simply be missing in the dwarfs, depending on the IMF and the total stellar mass (or mass concentration) in the dSph galaxies. 